\title{
An Analysis of Action Recognition Datasets for Language and Vision Tasks
}

\author{
Spandana Gella and Frank Keller \\ Institute for Language, Cognition and Computation \\ School of Informatics, University of Edinburgh \\ 10 Crichton Street, Edinburgh EH8 9AB \\ S.Gella@sms.ed.ac.uk, keller@inf.ed.ac.uk
}

\begin{abstract}
A large amount of recent research has focused on tasks that combine language and vision, resulting in a proliferation of datasets and methods. One such task is action recognition, whose applications include image annotation, scene understanding and image retrieval. In this survey, we categorize the existing approaches based on how they conceptualize this problem and provide a detailed review of existing datasets, highlighting their diversity as well as advantages and disadvantages. We focus on recently developed datasets which link visual information with linguistic resources and provide a fine-grained syntactic and semantic analysis of actions in images.
\end{abstract}

\section{Introduction}

Action recognition is the task of identifying the action being depicted in a video or still image. The task is useful for a range of applications such as generating descriptions, image/video retrieval, surveillance, and human-computer interaction. It has been widely studied in computer vision, often on videos (Nagel, 1994; Forsyth et al., 2005), where motion and temporal information provide cues for recognizing actions (Taylor et al., 2010). However, many actions are recognizable from still images, see the examples in Figure 1. Due to the absence of motion cues and temporal features (Ikizler et al., 2008) action recognition from stills is more challenging. Most of the existing work can be categorized into four tasks: (a) action classification (AC); (b) determining human-object interaction (HOI); (c) visual verb sense disambiguation (VSD); and (d) visual semantic role labeling (VSRL). In Figure 2 we illustrate each of these

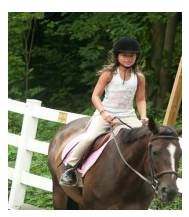

riding horse

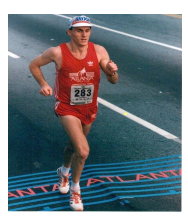

running

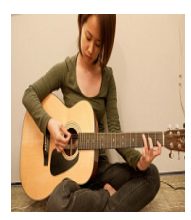

playing guitar

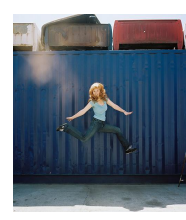

jumping
Figure 1: Examples of actions in still images

tasks and show how they are related to each other.

Until recently, action recognition was studied as action classification on small-scale datasets with a limited number of predefined actions labels (Ikizler et al., 2008; Gupta et al., 2009; Yao and FeiFei, 2010; Everingham et al., 2010; Yao et al., 2011). Often the labels in action classification tasks are verb phrases or a combination of verb and object such as playing baseball, riding horse. These datasets have helped in building models and understanding which aspects of an image are important for classifying actions, but most methods are not scalable to larger numbers of actions (Ramanathan et al., 2015). Action classification models are trained on images annotated with mutually exclusive labels, i.e., the assumption is that only a single label is relevant for a given image. This ignores the fact that actions such as holding bicycle and riding bicycle can co-occur in the same image. To address these issues and also to understand the range of possible interactions between humans and objects, the human-object interaction (HOI) detection task has been proposed, in which all possible interactions between a human and a given object have to be identified (Le et al., 2014; Chao et al., 2015; Lu et al., 2016).

However, both action classification and HOI detection do not consider the ambiguity that arises when verbs are used as labels, e.g., the verb play has multiple meanings in different contexts. On the other hand, action labels consisting of verbobject pairs can miss important generalizations: 


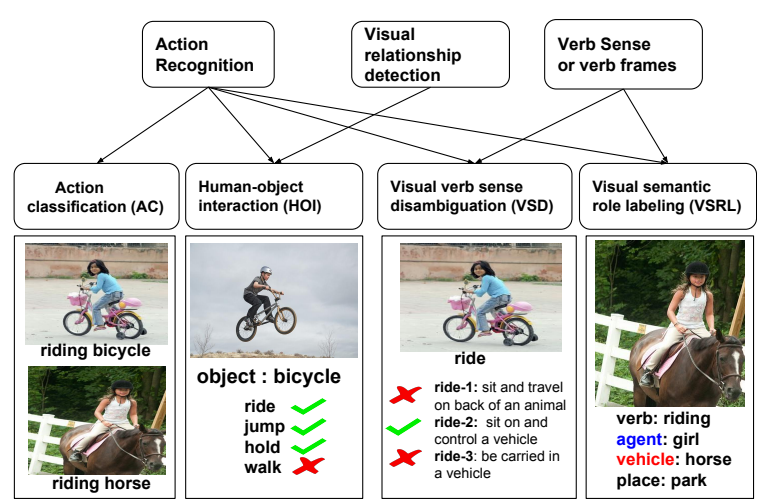

Figure 2: Categorization of action recognition tasks in images

riding horse and riding elephant both instantiate the same verb semantics, i.e., riding animal. Thirdly, existing action labels miss generalizations across verbs, e.g., the fact that fixing bike and repairing bike are semantically equivalent, in spite of the use of different verbs. These observations have led authors to argue that actions should be analyzed at the level of verb senses. Gella et al. (2016) propose the new task of visual verb sense disambiguation (VSD), in which a verbimage pair is annotated with a verb sense taken from an existing lexical database (OntoNotes in this case). While VSD handles distinction between different verb senses, it does not identify or localize the objects that participate in the action denoted by the verb. Recent work (Gupta and Malik, 2015; Yatskar et al., 2016) has filled this gap by proposing the task of visual semantic role labeling (VSRL), in which images are labeled with verb frames, and the objects that fill the semantic roles of the frame are identified in the image.

In this paper, we provide a unified view of action recognition tasks, pointing out their strengths and weaknesses. We survey existing literature and provide insights into existing datasets and models for action recognition tasks.

\section{Datasets for Action Recognition}

We give an overview of commonly used datasets for action recognition tasks in Table 1 and group them according to subtask. We observe that the number of verbs covered in these datasets is often smaller than the number of action labels reported (see Table 1, columns \#V and \#L) and in many cases the action label involves object reference. A few of the first action recognition datasets such as the Ikizler and Willow datasets (Ikizler et al.,
2008; Delaitre et al., 2010) had action labels such as throwing and running; they were taken from the sports domain and exhibited diversity in camera view point, background and resolution. Then datasets were created to capture variation in human poses in the sports domain for actions such as tennis serve and cricket bowling; typically features based on poses and body parts were used to build models (Gupta et al., 2009). Further datasets were created based on the intuition that object information helps in modeling action recognition (Li and Fei-Fei, 2007; Ikizler-Cinbis and Sclaroff, 2010), which resulted in the use of action labels such as riding horse or riding bike (Everingham et al., 2010; Yao et al., 2011). Not only were most of these datasets domain specific, but the labels were also manually selected and mutually exclusive, i.e., two actions cannot co-occur in the same image. Also, most of these datasets do not localize objects or identify their semantic roles.

\subsection{Identifying Visual Verbs and Verb Senses}

The limitations with early datasets (small scale, domain specificity, and the use of ad-hoc labels that combine verb and object) have been recently addressed in a number of broad-coverage datasets that offer linguistically motivated labels. Often these datasets use existing linguistic resources such as VerbNet (Schuler, 2005), OntoNotes (Hovy et al., 2006) and FrameNet (Baker et al., 1998) to classify verbs and their senses. This allows for a more general, semantically motivated treatment of verbs and verb phrases, and also takes into account that not all verbs are depictable. For example, abstract verbs such as presuming and $a c$ quiring are not depictable at all, while other verbs have both depictable and non-depictable senses: play is non-depictable in playing with emotions, but depictable in playing instrument and playing sport. The process of identifying depictable verbs or verb senses is used by Ronchi and Perona (2015), Gella et al. (2016) and Yatskar et al. (2016) to identify visual verbs, visual verb senses, and the semantic roles of the participating objects respectively. In all the cases the process of identifying visual verbs or senses is carried out by human annotators via crowd-sourcing platforms. Visualness labels for 935 OntoNotes verb senses corresponding to 154 verbs is provided by Gella et al. (2016), while Yatskar et al. (2016) provides visualness labels for 9683 FrameNet verbs. 


\begin{tabular}{|c|c|c|c|c|c|c|c|c|c|c|c|}
\hline Dataset & Task & \#L & $\# \mathrm{~V}$ & Obj & $\operatorname{Imgs}$ & Sen & Des & $\mathrm{Cln}$ & ML & Resource & Example Labels \\
\hline Willow (Delaitre et al., 2010) & $\mathrm{AC}$ & 7 & 6 & 5 & 986 & $\mathrm{~N}$ & $\mathrm{~N}$ & $\mathrm{Y}$ & $\mathrm{Y}$ & - & riding bike, photographing \\
\hline Stanford 40 Actions (Yao et al., 2011) & $\mathrm{AC}$ & 40 & 33 & 31 & $9.5 \mathrm{k}$ & $\mathrm{N}$ & $\mathrm{N}$ & $\mathrm{Y}$ & $\mathrm{N}$ & - & cut vegetables, ride horse \\
\hline PASCAL 2012 (Everingham et al., 2015) & $\mathrm{AC}$ & 11 & 9 & 6 & $4.5 \mathrm{k}$ & $\mathrm{N}$ & $\mathrm{N}$ & $\mathrm{Y}$ & $\mathrm{Y}$ & - & riding bike, riding horse \\
\hline 89 Actions (Le et al., 2013) & $\mathrm{AC}$ & 89 & 36 & 19 & $2 \mathrm{k}$ & $\mathrm{N}$ & $\mathrm{N}$ & $\mathrm{Y}$ & $\mathrm{N}$ & - & ride bike, fix bike \\
\hline COCO-a (Ronchi and Perona, 2015) & HOI & -1 & 140 & 80 & $10 \mathrm{k}$ & $\mathrm{N}$ & $\mathrm{Y}$ & $\mathrm{Y}$ & $\mathrm{Y}$ & VerbNet & walk bike, hold bike \\
\hline Google Images (Ramanathan et al., 2015) & $\mathrm{AC}$ & 2880 & - & - & $102 \mathrm{k}$ & $\mathrm{N}$ & $\mathrm{N}$ & $\mathrm{N}$ & $\mathrm{N}$ & - & riding horse, riding camel \\
\hline HICO (Chao et al., 2015) & HOI & 600 & 111 & 80 & $47 \mathrm{k}$ & $\mathrm{Y}$ & $\mathrm{N}$ & $\mathrm{Y}$ & $\mathrm{Y}$ & WordNet & ride\#v\#1 bike; hold\#v\#2 bike \\
\hline VCOCO-SRL (Gupta and Malik, 2015) & VSRL & & 26 & 48 & $10 \mathrm{k}$ & $\mathrm{N}$ & $\mathrm{Y}$ & $\mathrm{Y}$ & $\mathrm{Y}$ & - & verb: hit; instr: bat; obj: ball \\
\hline imSitu (Yatskar et al., 2016) & VSRL & -5 & 504 & $11 \mathrm{k}$ & $126 \mathrm{k}$ & $\mathrm{Y}$ & $\mathrm{N}$ & $\mathrm{Y}$ & $\mathrm{N}$ & $\begin{array}{l}\text { FrameNet } \\
\text { WordNet }\end{array}$ & $\begin{array}{l}\text { verb: ride; agent: girl\#n\#2 } \\
\text { vehicle: bike\#n\#1; } \\
\text { place: road\#n\#2 }\end{array}$ \\
\hline
\end{tabular}

Table 1: Comparison of various existing action recognition datasets. \#L denotes number of action labels in the dataset; \#V denotes number of verbs covered in the dataset; Obj indicates number of objects annotated; Sen indicates whether sense ambiguity is explicitly handled; Des indicates whether image descriptions are included; Cln indicates whether dataset is manually verified; ML indicates the possibility of multiple labels per image; Resource indicates linguistic resource used to label actions.

\subsection{Datasets Beyond Action Classification}

Over the last few years tasks that combine language and vision such as image description and visual question answering have gained much attention. This has led to the creation of new, large datasets such as MSCOCO (Chen et al., 2015) and the VQA dataset (Antol et al., 2015). Although these datasets are not created for action recognition, a number of attempts have been made to use the verbs present in image descriptions to annotate actions. The COCO-a, VerSe and VCOCO-SRL datasets all use the MSCOCO image descriptions to annotate fine-grained aspects of interaction and semantic roles.

HICO: The HICO dataset has $47.8 \mathrm{k}$ images annotated with 600 categories of human-object interactions with 111 verbs applying to 80 object categories of MSCOCO. It is annotated to include diverse interactions for objects and has an average of 6.5 distinct interactions per object category. Unlike other HOI datasets such as TUHOI which label interactions as verbs and ignore senses, the HOI categories of HICO are based on WordNet (Miller, 1995) verb senses. The HICO dataset also has multiple annotations per object and it incorporates the information that certain interactions such as riding a bike and holding a bike often co-occur. However, it fails to include annotations to distinguish between multiple senses of a verb.

Visual Genome: The dataset created by Krishna et al. (2016) has dense annotations of objects, at- tributes, and relationships between objects. The Visual Genome dataset contains 105k images with 40k unique relationships between objects. Unlike other HOI datasets such as HICO, visual genome relationships also include prepositions, comparative and prepositional phrases such as near and taller than, making the visual relationship task more generic than action recognition. Krishna et al. (2016) combine all the annotations of objects, relationships, and attributes into directed graphs known as scene graphs.

COCO-a: Ronchi and Perona (2015) present Visual VerbNet (VVN), a list of 140 common visual verbs manually mined from English VerbNet (Schuler, 2005). The coverage of visual verbs in this dataset is not complete, as many visual verbs such as dive, perform and shoot are not included. This also highlights a bias in this dataset as the authors relied on occurrence in MSCOCO as a verification step to consider a verb as visual. They annotated 10k images containing human subjects with one of the 140 visual verbs, for 80 MSCOCO objects. This dataset has better coverage of human-object interactions than the HICO dataset despite of missing many visual verbs.

VerSe: Gella et al. (2016) created a dataset of $3.5 \mathrm{k}$ images sampled from the MSCOCO and TUHOI datasets and annotated it with 90 verbs and their OntoNotes senses to distinguish different verb senses using visual context. This is the first dataset that aims to annotate all visual senses 
of a verb. However, the total number of images annotated and number of images for some senses is relatively small, which makes it difficult to use this dataset to train models. The authors further divided their 90 verbs into motion and non-motion verbs according to Levin (1993) verb classes and analyzed visual ambiguity in the task of visual sense disambiguation.

VCOCO-SRL: Gupta and Malik (2015) annotated a dataset of $16 \mathrm{k}$ person instances in $10 \mathrm{k}$ images with 26 verbs and associated objects in the scene with the semantic roles for each action. The main aim of the dataset is to build models for visual semantic role labeling in images. This task involves identifying the actions depicted in an image, along with the people and objects that instantiate the semantic roles of the actions. In the VCOCO-SRL dataset, each person instance is annotated with a mean of 2.8 actions simultaneously.

imSitu: Yatskar et al. (2016) annotated a large dataset of $125 \mathrm{k}$ images with 504 verbs, $1.7 \mathrm{k}$ semantic roles and $11 \mathrm{k}$ objects. They used FrameNet verbs, frames and associated objects or scenes with roles to develop the dataset. They annotate every image with a single verb and the semantic roles of the objects present in the image. VCOCOSRL the is dataset most similar to imSitu, however VCOCO-SRL includes localization information of agents and all objects and provides multiple action annotations per image. On the other hand, imSitu is the dataset that covers highest number of verbs, while also omitting many commonly studied polysemous verbs such as play.

\subsection{Diversity in Datasets}

With the exception of a few datasets such as COCO-a, VerSe, imSitu all action recognition datasets have manually picked labels or focus on covering actions in specific domains such as sports. Alternatively, many datasets only cover actions relevant to specific object categories such as musical instruments, animals and vehicles. In the real world, people interact with many more objects and perform actions relevant to a wide range of domains such as personal care, household activities, or socializing. This limits the diversity and coverage of existing action recognition datasets. Recently proposed datasets partly handle this issue by using generic linguistic resources to extend the vocabulary of verbs in action labels. The diversity issue has also been high- lighted and addressed in recent video action recognition datasets (Caba Heilbron et al., 2015; Sigurdsson et al., 2016), which include generic household activities. An analysis of various image description and question answering datasets by Ferraro et al. (2015) shows the bias in the distribution of word categories. Image description datasets have a higher distribution of nouns compared to other word categories, indicating that the descriptions are object specific, limiting their usefulness for action-based tasks.

\section{Relevant Language and Vision Tasks}

Template based description generation systems for both videos and images rely on identifying subject-verb-object triples and use language modeling to generate or rank descriptions (Yang et al., 2011; Thomason et al., 2014; Bernardi et al., 2016). Understanding actions also plays an important role in question answering, especially when the question is pertaining to an action depicted in the image. There are some specifically curated question answering datasets which target human activities or relationships between a pair of objects (Yu et al., 2015). Mallya and Lazebnik (2016) have shown that systems trained on action recognition datasets could be used to improve the accuracy of visual question answering systems that handle questions related to human activity and human-object relationships. Action recognition datasets could be used to learn actions that are visually similar such as interacting with panda and feeding a panda or tickling a baby and calming a baby, which cannot be learned from text alone (Ramanathan et al., 2015). Visual semantic role labeling is a crucial step for grounding actions in the physical world (Yang et al., 2016).

\section{Action Recognition Models}

Most of the models proposed for action classification and human-object interaction tasks rely on identifying higher-level visual cues present in the image, including human bodies or body parts (Ikizler et al., 2008; Gupta et al., 2009; Yao et al., 2011; Andriluka et al., 2014), objects (Gupta et al., 2009), and scenes (Li and Fei-Fei, 2007). Higherlevel visual cues are obtained through low-level features extracted from the image such as Scale Invariant Feature Transforms (SIFT), Histogram of Oriented Gradients (HOG), and Spatial Envelopes (Gist) features (Lowe, 1999; Dalal and Triggs, 
2005). These are useful in identifying key points, detecting humans, and scene or background information in images, respectively. In addition to identifying humans and objects, the relative position or angle between a human and an object is useful in learning human-object interactions (Le et al., 2014). Most of the existing approaches rely on learning supervised classifiers over low-level features to predict action labels.

More recent approaches are based on end-toend convolutional neural network architectures which learn visual cues such as objects and image features for action recognition (Chao et al., 2015; Zhou et al., 2016; Mallya and Lazebnik, 2016). While most of the action classification models rely solely on visual information, models proposed for human-object interaction or visual relationship detection sometimes combine human and object identification (using visual features) with linguistic knowledge (Le et al., 2014; Krishna et al., 2016; Lu et al., 2016). Other work on identifying actions, especially methods that focus on relationships that are infrequent or unseen, utilize word vectors learned on large text corpora as an additional source of information ( $\mathrm{Lu}$ et al., 2016). Similarly, Gella et al. (2016) show that embeddings generated from textual data associated with images (object labels, image descriptions) is useful for visual verb sense disambiguation, and is complementary to visual information.

\section{Discussion}

Linguistic resources such as WordNet, OntoNotes, and FrameNet play a key role in textual sense disambiguation and semantic role labeling. The visual action disambiguation and visual semantic role labeling tasks are extensions of their textual counterparts, where context is provided as an image instead of as text. Linguistic resources therefore have to play a key role if we are to make rapid progress in these language and vision tasks. However, as we have shown in this paper, only a few of the existing datasets for action recognition and related tasks are based on linguistic resources (Chao et al., 2015; Gella et al., 2016; Yatskar et al., 2016). This is despite the fact that the WordNet noun hierarchy (for example) has played an important role in recent progress in object recognition, by virtue of underlying the ImageNet database, the de-facto standard for this task (Russakovsky et al., 2015). The success of ImageNet for objects has in turn helped NLP tasks such as bilingual lexicon induction (Vulić et al., 2016). In our view, language and vision datasets that are based on the WordNet, OntoNotes, or FrameNet verb sense inventories can play a similar role for tasks such as action recognition or visual semantic role labeling, and ultimately be useful also for more distantly related tasks such as language grounding.

Another argument for linking language and vision datasets with linguistic resources is that this enables us to deploy the datasets in a multilingual setting. For example a polysemous verb such as ride in English has multiple translations in German and Spanish, depending on the context and the objects involved. Riding a horse is translated as reiten in German and cabalgar in Spanish, whereas riding a bicycle is translated as fahren in German and pedalear in Spanish. In contrast, some polysemous verb (e.g., English play) are always translated as the same verb, independent of sense (spielen in German). Such sense mappings are discoverable from multilingual lexical resources (e.g., BabelNet, Navigli and Ponzetto 2010), which makes it possible to construct language and vision models that are applicable to multiple languages. This opportunity is lost if language and vision dataset are constructed in isolation, instead of using existing linguistic resources.

\section{Conclusions}

In this paper, we have shown the evolution of action recognition datasets and tasks from simple ad-hoc labels to the fine-grained annotation of verb semantics. It is encouraging to see the recent increase in datasets that deal with sense ambiguity and annotate semantic roles, while using standard linguistic resources. One major remaining issue with existing datasets is their limited coverage, and the skewed distribution of verbs or verb senses. Another challenge is the inconsistency in annotation schemes and task definitions across datasets. For example Chao et al. (2015) used WordNet senses as interaction labels, while Gella et al. (2016) used the more coarsegrained OntoNotes senses. Yatskar et al. (2016) used FrameNet frames for semantic role annotation, while Gupta and Malik (2015) used manually curated roles. If we are to develop robust, domain independent models, then we need to standardize annotation schemes and use the same linguistic resources across datasets. 


\section{References}

Mykhaylo Andriluka, Leonid Pishchulin, Peter Gehler, and Bernt Schiele. 2014. 2d human pose estimation: New benchmark and state of the art analysis. In Proceedings of the IEEE Conference on Computer Vision and Pattern Recognition. pages 3686-3693.

Stanislaw Antol, Aishwarya Agrawal, Jiasen Lu, Margaret Mitchell, Dhruv Batra, C. Lawrence Zitnick, and Devi Parikh. 2015. VQA: visual question answering. In 2015 IEEE International Conference on Computer Vision, ICCV 2015, Santiago, Chile, December 7-13, 2015. pages 2425-2433.

Collin F Baker, Charles J Fillmore, and John B Lowe. 1998. The berkeley framenet project. In Proceedings of the 36th Annual Meeting of the Association for Computational Linguistics and 17th International Conference on Computational LinguisticsVolume 1. Association for Computational Linguistics, pages 86-90.

Raffaella Bernardi, Ruket Cakici, Desmond Elliott, Aykut Erdem, Erkut Erdem, Nazli Ikizler-Cinbis, Frank Keller, Adrian Muscat, and Barbara Plank. 2016. Automatic description generation from images: A survey of models, datasets, and evaluation measures. Journal of Artifical Intelligence Research 55:409-442.

Fabian Caba Heilbron, Victor Escorcia, Bernard Ghanem, and Juan Carlos Niebles. 2015. Activitynet: A large-scale video benchmark for human activity understanding. In Proceedings of the IEEE Conference on Computer Vision and Pattern Recognition. pages 961-970.

Yu-Wei Chao, Zhan Wang, Yugeng He, Jiaxuan Wang, and Jia Deng. 2015. HICO: A benchmark for recognizing human-object interactions in images. In 2015 IEEE International Conference on Computer Vision, ICCV 2015, Santiago, Chile, December 7-13, 2015. pages 1017-1025.

Xinlei Chen, Hao Fang, Tsung-Yi Lin, Ramakrishna Vedantam, Saurabh Gupta, Piotr Dollár, and C. Lawrence Zitnick. 2015. Microsoft COCO captions: Data collection and evaluation server. CoRR abs/1504.00325.

Navneet Dalal and Bill Triggs. 2005. Histograms of oriented gradients for human detection. In Computer Vision and Pattern Recognition, 2005. CVPR 2005. IEEE Computer Society Conference on. IEEE, volume 1, pages 886-893.

Vincent Delaitre, Ivan Laptev, and Josef Sivic. 2010. Recognizing human actions in still images: a study of bag-of-features and part-based representations. In BMVC 2010-21 st British Machine Vision Conference.

Mark Everingham, S. M. Ali Eslami, Luc Van Gool, Christopher K. I. Williams, John M. Winn, and Andrew Zisserman. 2015. The Pascal visual object classes challenge: A retrospective. International Journal of Computer Vision 111(1):98-136.

Mark Everingham, Luc J. Van Gool, Christopher K. I. Williams, John M. Winn, and Andrew Zisserman. 2010. The Pascal visual object classes (VOC) challenge. International Journal of Computer Vision 88(2):303-338.

Francis Ferraro, Nasrin Mostafazadeh, Ting-Hao (Kenneth) Huang, Lucy Vanderwende, Jacob Devlin, Michel Galley, and Margaret Mitchell. 2015. A survey of current datasets for vision and language research. In Proceedings of the 2015 Conference on Empirical Methods in Natural Language Processing, EMNLP 2015, Lisbon, Portugal, September 1721, 2015. pages 207-213.

David A. Forsyth, Okan Arikan, Leslie Ikemoto, James F. O'Brien, and Deva Ramanan. 2005. Computational studies of human motion: Part 1, tracking and motion synthesis. Foundations and Trends in Computer Graphics and Vision 1(2/3).

Spandana Gella, Mirella Lapata, and Frank Keller. 2016. Unsupervised visual sense disambiguation for verbs using multimodal embeddings. In Proceedings of the 2016 Conference on North American Chapter of the Association for Computational Linguistics: Human Language Technologies, San Diego California, USA, June 12-17, 2016. pages 182-192.

Abhinav Gupta, Aniruddha Kembhavi, and Larry S. Davis. 2009. Observing human-object interactions: Using spatial and functional compatibility for recognition. IEEE Transactions on Pattern Analysis and Machine Intelligence 31(10):1775-1789.

Saurabh Gupta and Jitendra Malik. 2015. Visual semantic role labeling. CoRR abs/1505.04474.

Eduard H. Hovy, Mitchell P. Marcus, Martha Palmer, Lance A. Ramshaw, and Ralph M. Weischedel. 2006. Ontonotes: The $90 \%$ solution. In $\mathrm{Hu}$ man Language Technology Conference of the North American Chapter of the Association of Computational Linguistics, Proceedings, June 4-9, 2006, New York, New York, USA. pages 57-60.

Nazli Ikizler, Ramazan Gokberk Cinbis, Selen Pehlivan, and Pinar Duygulu. 2008. Recognizing actions from still images. In 19th International Conference on Pattern Recognition (ICPR 2008), December 811, 2008, Tampa, Florida, USA. pages 1-4.

Nazli Ikizler-Cinbis and Stan Sclaroff. 2010. Object, scene and actions: Combining multiple features for human action recognition. In European conference on computer vision. Springer, pages 494-507.

Ranjay Krishna, Yuke Zhu, Oliver Groth, Justin Johnson, Kenji Hata, Joshua Kravitz, Stephanie Chen, Yannis Kalantidis, Li-Jia Li, David A Shamma, et al. 2016. Visual genome: Connecting language and vision using crowdsourced dense image annotations. arXiv preprint arXiv:1602.07332 . 
Dieu Thu Le, Raffaella Bernardi, and Jasper Uijlings. 2013. Exploiting language models to recognize unseen actions. In Proceedings of the 3rd ACM conference on International conference on multimedia retrieval. ACM, pages 231-238.

Dieu-Thu Le, Jasper Uijlings, and Raffaella Bernardi. 2014. Proceedings of the Third Workshop on Vision and Language, Dublin City University and the Association for Computational Linguistics, chapter TUHOI: Trento Universal Human Object Interaction Dataset, pages 17-24.

Beth Levin. 1993. English verb classes and alternations: A preliminary investigation. University of Chicago Press.

Li-Jia Li and Li Fei-Fei. 2007. What, where and who? classifying events by scene and object recognition. In Computer Vision, 2007. ICCV 2007. IEEE 11th International Conference on. IEEE, pages 1-8.

David G Lowe. 1999. Object recognition from local scale-invariant features. In Computer vision, 1999. The proceedings of the seventh IEEE international conference on. Ieee, volume 2, pages 1150-1157.

Cewu Lu, Ranjay Krishna, Michael Bernstein, and Li Fei-Fei. 2016. Visual relationship detection with language priors. In European Conference on Computer Vision. Springer, pages 852-869.

Arun Mallya and Svetlana Lazebnik. 2016. Learning models for actions and person-object interactions with transfer to question answering. In $E u$ ropean Conference on Computer Vision. Springer, pages $414-428$.

George A Miller. 1995. Wordnet: a lexical database for english. Communications of the ACM 38(11):3941 .

Hans-Hellmut Nagel. 1994. A vision of "vision and language" comprises action: An example from road traffic. Artif. Intell. Rev. 8(2-3):189-214.

Roberto Navigli and Simone Paolo Ponzetto. 2010. Babelnet: Building a very large multilingual semantic network. In ACL 2010, Proceedings of the 48th Annual Meeting of the Association for Computational Linguistics, July 11-16, 2010, Uppsala, Sweden. pages 216-225.

Vignesh Ramanathan, Congcong Li, Jia Deng, Wei Han, Zhen Li, Kunlong Gu, Yang Song, Samy Bengio, Chuck Rossenberg, and Li Fei-Fei. 2015. Learning semantic relationships for better action retrieval in images. In Proceedings of the IEEE Conference on Computer Vision and Pattern Recognition. pages 1100-1109.

Matteo Ruggero Ronchi and Pietro Perona. 2015. Describing common human visual actions in images. In Proceedings of the British Machine Vision Conference (BMVC 2015). BMVA Press, pages 52.152.12 .
Olga Russakovsky, Jia Deng, Hao Su, Jonathan Krause, Sanjeev Satheesh, Sean Ma, Zhiheng Huang, Andrej Karpathy, Aditya Khosla, Michael S. Bernstein, Alexander C. Berg, and Fei-Fei Li. 2015. Imagenet large scale visual recognition challenge. International Journal of Computer Vision 115(3):211-252.

Karin Kipper Schuler. 2005. VerbNet: A broadcoverage, comprehensive verb lexicon. Ph.D. thesis, University of Pennsylvania.

Gunnar A Sigurdsson, Gül Varol, Xiaolong Wang, Ali Farhadi, Ivan Laptev, and Abhinav Gupta. 2016. Hollywood in homes: Crowdsourcing data collection for activity understanding. In European Conference on Computer Vision. Springer, pages 510-526.

Graham W Taylor, Rob Fergus, Yann LeCun, and Christoph Bregler. 2010. Convolutional learning of spatio-temporal features. In European conference on computer vision. Springer, pages 140-153.

Jesse Thomason, Subhashini Venugopalan, Sergio Guadarrama, Kate Saenko, and Raymond J. Mooney. 2014. Integrating language and vision to generate natural language descriptions of videos in the wild. In COLING 2014, 25th International Conference on Computational Linguistics, Proceedings of the Conference: Technical Papers, August 23-29, 2014, Dublin, Ireland. pages 1218-1227.

Ivan Vulić, Douwe Kiela, Stephen Clark, and MarieFrancine Moens. 2016. Multi-modal representations for improved bilingual lexicon learning. In Proceedings of the 54th Annual Meeting of the Association for Computational Linguistics. ACL, pages 188-194.

Shaohua Yang, Qiaozi Gao, Changsong Liu, Caiming Xiong, Song-Chun Zhu, and Joyce Y. Chai. 2016. Grounded semantic role labeling. In NAACL HLT 2016, The 2016 Conference of the North American Chapter of the Association for Computational Linguistics: Human Language Technologies, San Diego California, USA, June 12-17, 2016. pages 149-159.

Yezhou Yang, Ching Lik Teo, Hal Daumé III, and Yiannis Aloimonos. 2011. Corpus-guided sentence generation of natural images. In Proceedings of the Conference on Empirical Methods in Natural Language Processing. Association for Computational Linguistics, pages 444-454.

Bangpeng Yao and Li Fei-Fei. 2010. Grouplet: A structured image representation for recognizing human and object interactions. In Computer Vision and Pattern Recognition (CVPR), 2010 IEEE Conference on. IEEE, pages 9-16.

Bangpeng Yao, Xiaoye Jiang, Aditya Khosla, Andy Lai Lin, Leonidas Guibas, and Li Fei-Fei. 2011. Human action recognition by learning bases of action attributes and parts. In Computer Vision (ICCV), 2011 IEEE International Conference on. IEEE, pages 1331-1338. 
Mark Yatskar, Luke Zettlemoyer, and Ali Farhadi. 2016. Situation recognition: Visual semantic role labeling for image understanding. In 2016 IEEE Conference on Computer Vision and Pattern Recognition, CVPR 2016, Las Vegas, NV, USA, June 26July 1, 2016.

Licheng Yu, Eunbyung Park, Alexander C. Berg, and Tamara L. Berg. 2015. Visual madlibs: Fill in the blank description generation and question answering. In 2015 IEEE International Conference on Computer Vision, ICCV 2015, Santiago, Chile, December 7-13, 2015. pages 2461-2469.

Bolei Zhou, Aditya Khosla, Agata Lapedriza, Aude Oliva, and Antonio Torralba. 2016. Learning deep features for discriminative localization. In Proceedings of the IEEE Conference on Computer Vision and Pattern Recognition. pages 2921-2929. 